\title{
A COMBINATORIAL FORMULA FOR NORMAL STIEFEL-WHITNEY CLASSES ${ }^{1}$
}

\author{
T. BANCHOFF AND C. MCCRORY
}

\begin{abstract}
Using an ordering of the vertices of a combinatorial $n$-manifold $K$, we give an explicit description of a simplicial mod 2 cycle $c_{n-i}(K)$ which represents the dual of the $i$ th normal Stiefel-Whitney class of $K$.
\end{abstract}

1. The formula. A local ordering of a simplical complex $K$ is a partial ordering of the vertices of $K$ which restricts to a total order on the closed star of each vertex. If $K$ is a locally ordered $n$-dimensional complex, and $(\sigma, \tau)$ is an ordered pair of $n$-simplices of $K$ such that $\sigma \cap \tau \neq \varnothing$, define $\mu(\sigma, \tau) \in$ $\mathrm{Z} / 2 \mathbf{Z}$ as follows. Since $\sigma \cap \tau \neq \varnothing$, there is a vertex $v$ such that $\sigma$ and $\tau$ are in $\operatorname{star}(v)$. Let $v_{1}, \ldots, v_{s}$ be the vertices of $\sigma$ and $\tau$ in the given total order of $\operatorname{star}(v)$. (If a vertex belongs to both $\sigma$ and $\tau$, it should be listed only once.) Let $\mu(\sigma, \tau)=1$ if $v_{1}, v_{3}, v_{5}, \ldots$ are in $\sigma$ and $v_{2}, v_{4}, v_{6}, \ldots$ are in $\tau$, and $\mu(\sigma, \tau)$ $=0$ otherwise. For $i=1,2, \ldots, n$, let $c_{n-i}(K)$ be the simplicial $\bmod 2$ $(n-i)$-chain $\Sigma \mu(\sigma, \tau) \sigma \cap \tau$, summed over all ordered pairs $(\sigma, \tau)$ of $n$-simplices such that $\operatorname{dim}(\sigma \cap \tau)=n-i$.

THEOREM 1. Let $K$ be a finite locally ordered combinatorial $n$-manifold without boundary. The chain $c_{n-i}(K)$ is a mod 2 cycle, and its homology class is Poincaré dual to $\bar{w}_{i}(K)$, the ith normal Stiefel-Whitney class of $K$.

A theorem of Levitt and Rourke [6] asserts that some combinatorial formula using a local ordering exists for every characteristic class of combinatorial manifolds. The prototype is the combinatorial formula for the tangential Stiefel-Whitney classes $w_{i}$ (cf. [4]). A generalization of this formula for $w_{i}$ has been given by Goldstein and Turner [3]. The observation that the Goldstein-Turner formula can be proved using our geometric definition of Stiefel-Whitney classes ([2], [7]) led us to the formula for $\bar{w}_{i}$ presented here. Its form was simplified by a remark of Lee Rudolph.

2. An example. Let $K$ be the triangulation of the projective plane obtained by identifying opposite points of the icosahedron. Order the vertices of $K$ as in Figure 1.

Presented to the Society, January 27, 1977; received by the editors April 13, 1978. AMS (MOS) subject classifications (1970). Primary 57D20, 55G05; Secondary 57C35.

Key words and phrases. Stiefel-Whitney class, combinatorial manifold, singularity cycle, homology operation.

${ }^{1}$ Supported by NSF grants MCS76-06324 and MCS76-09817. 


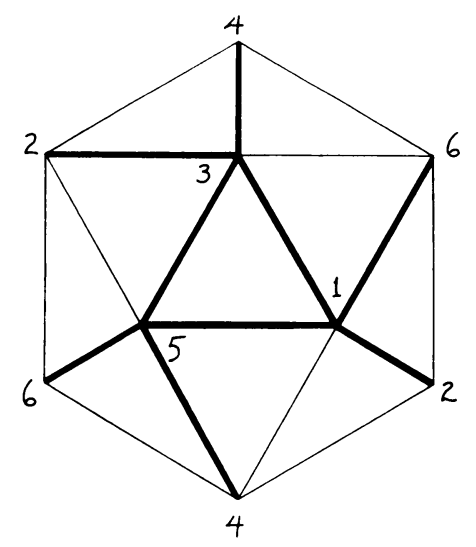

FIGURE 1

The cycle $c_{1}(K)$ is the sum of the nine edges emphasized in the figure. This cycle represents the nonzero class of $H_{1}(K)$ (mod 2 coefficients). For example, consider the edge $\langle 1,5\rangle$. The two 2 -simplices incident to it are $\langle 1,4,5\rangle$ and $\langle 1,3,5\rangle$. Since $\mu(\langle 1,4,5\rangle,\langle 1,3,5\rangle)=1$, the coefficient of $\langle 1,5\rangle$ in $c_{1}(K)$ is 1 .

The cycle $c_{0}(K)$ is the sum of the vertices $\langle 3\rangle$ and $\langle 6\rangle$, so its homology class is 0 . For example $\mu(\langle 1,3,5\rangle,\langle 1,2,4\rangle)=1, \mu(\langle 1,3,6\rangle,\langle 1,2,4\rangle)=1$, and $\mu(\sigma, \tau)=0$ for all other ordered pairs of 2-simplices $(\sigma, \tau)$ in the star of $\langle 1\rangle$. Therefore the coefficient of $\langle 1\rangle$ in $c_{0}(K)$ is 0 .

Now let $f: K \rightarrow R^{2}$ be a simplexwise-linear map which puts the vertices of $K$ in order along the parabola $\left(t, t^{2}\right)$. The cycle $c_{1}(K)$ is equal to the "fold cycle" of $f$. Figure 2 shows the image of the star of the edge $\langle 1,5\rangle$ under such a map.

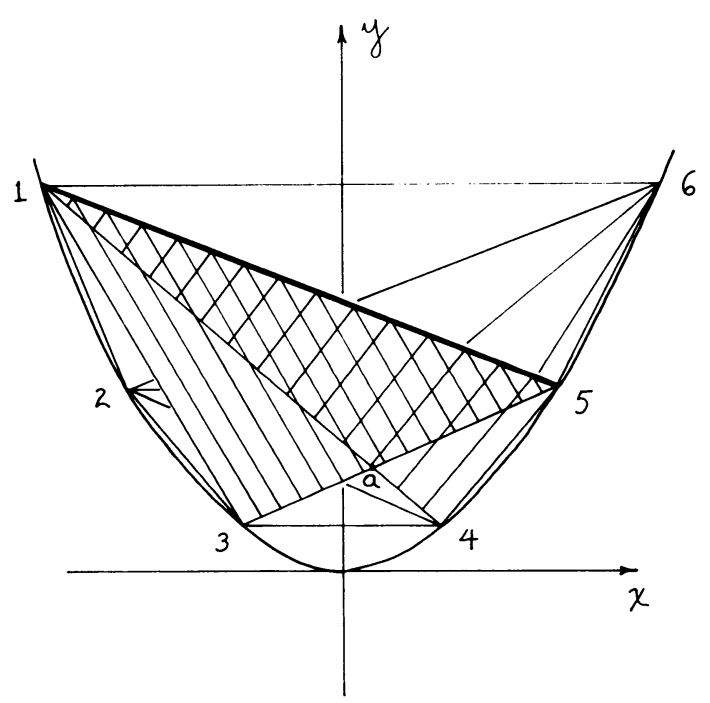

FIGURE 2 
Similarly, let $g: K \rightarrow R^{3}$ be a simplexwise-linear map which puts the vertices of $K$ in order along the twisted cubic $\left(t, t^{2}, t^{3}\right)$. The cycle $c_{0}(K)$ is equal to the "branch cycle" of $g$. In other words, if $\sigma$ and $\tau$ are 2-simplices such that $\sigma \cap \tau$ is a vertex, then

$$
g\left(\sigma^{\circ}\right) \cap g\left(\tau^{\circ}\right) \neq \varnothing
$$

if and only if either $\mu(\sigma, \tau)=1$ or $\mu(\tau, \sigma)=1$. ( $\left(\sigma^{\circ}\right.$ denotes the interior of $\sigma$.) Figure 3 shows the image of the union of $\langle 1,3,5\rangle$ and $\langle 1,2,4\rangle$ under such a map.

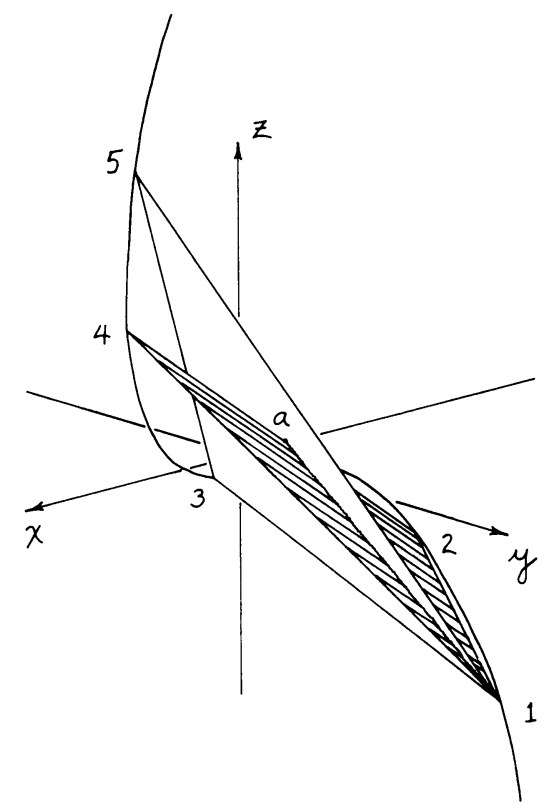

FiguRE 3

We will prove Theorem 1 by showing that, for any locally ordered $n$-complex $K$, the chain $c_{n-i}(K)$ is the branch chain of some map from $K$ to $R^{n+i-1}$.

3. The branch cycle. If $f: X \rightarrow Y$ is a continuous map, let $B(f)$ be the set of points $x \in X$ such that for every neighborhood $U$ of $x$ there exist $y$ and $z$ in $U$ with $y \neq z$ and $f(y)=f(z)$. The closed set $B(f)$ is the branch locus of $f$. The branch locus of $f$ is empty if and only if $f$ is locally injective. For example, if $f: N^{n} \rightarrow P^{p}$ is a stable differentiable map of smooth manifolds, with $n<p$, then $B(f)$ is precisely the singularity set of $f$ (cf. [7], [1]).

Let $f: K^{n} \rightarrow R^{p}$ be a simplexwise-linear map of the $n$-complex $K$ to Euclidean $p$-space, $n \leqslant p$. The map $f$ is general if $\operatorname{dim} f(\sigma)=\operatorname{dim} \sigma$ for all simplices $\sigma$ of $K$, and

$$
\operatorname{dim}\left(f\left(\sigma^{\circ}\right) \cap f\left(\tau^{\circ}\right)\right)=\operatorname{dim} \sigma+\operatorname{dim} \tau-p
$$

for all pairs of distinct simplices $\sigma, \tau$ of $K$ with $f\left(\sigma^{\circ}\right) \cap f\left(\tau^{\circ}\right) \neq \varnothing$. (No restriction is made on the dimension of intersection of three or more sim- 
plices.) If the span of $\left\{f\left(v_{0}\right), \ldots, f\left(v_{p}\right)\right\}$ is $R^{p}$ for every set of $p+1$ distinct vertices $v_{0}, \ldots, v_{p}$ of $K$, then $f$ is general.

If $f: K^{n} \rightarrow R^{p}$ is a general simplexwise-linear map, $n \leqslant p$, a simplicial $\bmod 2(2 n-p-1)$-chain $\mathscr{B}(f)$ in $K$, with support contained in $B(f)$, is defined as follows. The coefficient of the $(2 n-p-1)$-simplex $\omega$ in the chain $\Re(f)$ is the number $(\bmod 2)$ of unordered pairs of $n$-simplices $\{\sigma, \tau\}$ in the star of $\omega$ such that $f\left(\sigma^{\circ}\right) \cap f\left(\tau^{\circ}\right) \neq \varnothing$ (cf. Figures 2 and 3). The chain $\Re(f)$ is the branch chain of $f$.

THEOREM 2. Let $f: K^{n} \rightarrow R^{n+i-1}$ be a general simplexwise-linear map of the finite combinatorial $n$-manifold $K^{n}$ to Euclidean $(n+i-1)$-space, $i \geqslant 1$. The branch chain $\mathscr{B}(f)$ is a mod 2 cycle, and its homology class is Poincaré dual to $\bar{w}_{i}(K)$.

This theorem was conjectured in [7] and proved in [8], [9], and [5], in increasingly general settings. Here is a summary of the proof. If $K$ is a finite purely $n$-dimensional complex with $\bmod 2$ boundary $\partial K$, and $f: K \rightarrow R^{n+i-1}$ $(i \geqslant 1)$ is general, then $\partial \mathscr{B}(f)$ lies in $\partial K$, and the class of $\mathscr{B}(f)$ in $H_{n-i}(K, \partial K)$ is independent of $f$. Let $\Re^{i}(K)$ be this class. The function which assigns the class $\Re^{i}(K)$ to the complex $K$ is a geometric homology operation of degree $i$ which is uniquely characterized by a self-intersection property (cf. [9], [5]). Therefore $\mathscr{B}^{i}(K)=\mathrm{Sq}^{i}[K]$, where $\mathrm{Sq}^{i}$ is the $i$ th dual Steenrod homology operation and $[K] \in H_{n}(K, \partial K)$ is the fundamental class. Thom's formula for Stiefel-Whitney classes implies that $\mathrm{Sq}^{i}$ of the fundamental class of a manifold is Poincaré dual to $\bar{w}_{i}$.

To prove Theorem 1 it is enough to show that a local ordering of the vertices of an $n$-complex $K^{n}$ can be used to define a general map $f$ : $K^{n} \rightarrow R^{n+i-1}$ such that $c_{n-i}(K)=\mathscr{B}(f)$.

Let $K$ be an $n$-complex. Given a local ordering of the vertices of $K$ and an integer $p \geqslant n$, a moment map $f: K^{n} \rightarrow R^{p}$ is a simplexwise-linear map which puts the vertices of $K$ in order along the moment curve $\left(t, t^{2}, \ldots, t^{p}\right)$. That is, if $v_{i}$ is a vertex of $K$, then

$$
f\left(v_{i}\right)=\left(t_{i}, t_{i}^{2}, \ldots, t_{i}^{p}\right)
$$

where $t_{i}<t_{j}$ if $v_{i}<v_{j}$ in the given ordering.

LeMma 1. Any moment map is general.

Proof. If $f: K^{n} \rightarrow R^{p}$ is a moment map and $v_{0}, \ldots, v_{p}$ are distinct vertices of $K$, then the span of $\left\{f\left(v_{0}\right), \ldots, f\left(v_{p}\right)\right\}$ is $R^{p}$ if and only if the matrix

$$
\left[\begin{array}{ccccc}
1 & t_{0} & t_{0}^{2} & \ldots & t_{0}^{p} \\
\vdots & \vdots & \vdots & & \vdots \\
1 & t_{p} & t_{p}^{2} & \ldots & t_{p}^{p}
\end{array}\right]
$$

has rank $p$. But the determinant of this matrix is $\Pi_{i>j}\left(t_{i}-t_{j}\right) \neq 0$. 
Lemma 2 (A. Shapiro [10, p. 260]). Let $A$ and $B$ be simplices in $R^{p}$ with distinct vertices lying on the moment curve $C$ and $\operatorname{dim} A+\operatorname{dim} B=p$. Then $A \cap B \neq \varnothing$ if and only if the vertices of $A$ and $B$ alternate along $C$.

Note that the alternation of vertices implies that $|\operatorname{dim} A-\operatorname{dim} B| \leqslant 1$.

LEMMA 3. Let $f: K^{n} \rightarrow R^{p}$ be a general simplexwise-linear map, and let $\sigma, \tau$ be $n$-simplices of $K$ such that $\omega=\sigma \cap \tau$ is a $(2 n-p-1)$-simplex. Then $f\left(\sigma^{\circ}\right) \cap f\left(\tau^{\circ}\right) \neq \varnothing$ if and only if there are faces $\sigma^{\prime}<\sigma$ and $\tau^{\prime}<\tau$ such that $\sigma^{\prime} \cap \tau^{\prime}=\varnothing, \operatorname{dim} \sigma^{\prime}+\operatorname{dim} \tau^{\prime}=p$, and

$$
f\left(\left(\sigma^{\prime}\right)^{\circ}\right) \cap f\left(\left(\tau^{\prime}\right)^{\circ}\right) \neq \varnothing .
$$

Moreover, $\sigma^{\prime}$ and $\tau^{\prime}$ are unique, and $f(\sigma) \cap f(\tau)$ is the cone over $f(\omega)$ from a, where

$$
\{a\}=f\left(\left(\sigma^{\prime}\right)^{\circ}\right) \cap f\left(\left(\tau^{\prime}\right)^{\circ}\right)
$$

(cf. Figures 2 and 3).

Proof. For any simplex $\rho$ of $K$, let $v(\rho)$ be the set of vertices of $\rho$. If $\sigma^{\prime}$ and $\tau^{\prime}$ have the stated properties, then $v\left(\sigma^{\prime}\right) \cup v\left(\tau^{\prime}\right)=v(\sigma) \cup v(\tau)$. For $v\left(\sigma^{\prime}\right) \cup$ $v\left(\tau^{\prime}\right) \subset v(\sigma) \cup v(\tau)$, and both these sets have $p+2$ elements. Therefore $v(\omega) \cup v\left(\sigma^{\prime}\right)=v(\sigma)$, since $v(\omega) \cup v\left(\sigma^{\prime}\right) \subset v(\sigma) \subset v(\sigma) \cap\left(v\left(\sigma^{\prime}\right) \cup v\left(\tau^{\prime}\right)\right)=$ $\left(v(\sigma) \cap v\left(\tau^{\prime}\right)\right) \cup v\left(\sigma^{\prime}\right) \subset v(\omega) \cup v\left(\sigma^{\prime}\right)$. Similarly $v(\omega) \cup v\left(\tau^{\prime}\right)=v(\tau)$. Now let $a_{1} \in\left(\sigma^{\prime}\right)^{\circ}$ and $a_{2} \in\left(\tau^{\prime}\right)^{\circ}$ with $f\left(a_{1}\right)=f\left(a_{2}\right)=a \in f\left(\left(\sigma^{\prime}\right)^{\circ}\right) \cap f\left(\left(\tau^{\prime}\right)^{\circ}\right)$, and let $b \in \omega^{\circ}$. Then $\left(a_{1}+b\right) / 2 \in \sigma^{\circ}$ since $\sigma^{\prime}$ and $\omega$ span $\sigma$. Similarly $\left(a_{2}+b\right) / 2 \in$ $\tau^{\circ}$. So $f\left(\left(a_{1}+b\right) / 2\right)=f\left(\left(a_{2}+b\right) / 2\right)=(a+f(b)) / 2 \in f\left(\sigma^{\circ}\right) \cap f\left(\tau^{\circ}\right)$, and $f\left(\sigma^{\circ}\right) \cap f\left(\tau^{\circ}\right) \neq \varnothing$. (Note that the cone from $a$ over $f(\omega)$ is contained in $f(\sigma) \cap f(\tau)$.)

Conversely, suppose that $f\left(\sigma^{\circ}\right) \cap f\left(\tau^{\circ}\right) \neq \varnothing$. Now $f(\sigma) \cap f(\tau)$ is a convex cell in $R^{p}$ containing $f(\omega)$. Furthermore $f(\sigma) \cap f(\tau)$ is the union of the cells of a complex $C$. The open cells of $C$ are the sets $f\left(s^{\circ}\right) \cap f\left(t^{\circ}\right)$ for $s<\sigma$ and $t<\tau$. (Clearly $f(\sigma) \cap f(\tau)$ is the disjoint union of the open cells $f\left(s^{\circ}\right) \cap f\left(t^{\circ}\right)$, and the boundary of the closed cell $f(s) \cap f(t)$ is the union of $f\left(\left(s^{\prime}\right)^{\circ}\right) \cap$ $f\left(\left(t^{\prime}\right)^{\circ}\right)$ for $s^{\prime}<s$ and $t^{\prime}<t,\left(s^{\prime}, t^{\prime}\right) \neq(s, t)$.) The open cells of $C$ which are contained in $f(\omega)$ are the cells $f\left(w^{\circ}\right)$ with $w<\omega$, i.e. the cells $f\left(s^{\circ}\right) \cap f\left(t^{\circ}\right)$ with $s=t$.

Now suppose $s\left\langle\sigma\right.$ and $t\left\langle\tau\right.$ are such that $s \neq t$ and $\left.\operatorname{dim}\left(f\left(s^{\circ}\right) \cap f\left(t^{\circ}\right)\right)\right\rangle$ 1. Since $f$ is general, this implies $\operatorname{dim} s+\operatorname{dim} t \geqslant p+1$. Since $v(\sigma) \cup v(\tau)$ has $p+2$ elements, it follows that $s \cap t \neq \varnothing$. Thus the cell $f(s) \cap f(t)$ has some face in the cell $f(\omega)$. So each edge of the complex $C$ has at least one vertex in $f(\omega)$. Therefore $C$ has a unique vertex $a \notin f(\omega)$, and the convex cell $f(\sigma) \cap f(\tau)$ is the cone from $a$ over $f(\omega)$. Let $\{a\}=f\left(\left(\sigma^{\prime}\right)^{\circ}\right) \cap f\left(\left(\tau^{\prime}\right)^{\circ}\right)$ for $\sigma^{\prime}<\sigma$ and $\tau^{\prime}<\tau$. Since $f$ is general, $\operatorname{dim} \sigma^{\prime}+\operatorname{dim} \tau^{\prime}=p$, so $v\left(\sigma^{\prime}\right) \cup v\left(\tau^{\prime}\right)=$ $v(\sigma) \cup v(\tau)$ and $\sigma^{\prime} \cap \tau^{\prime}=\varnothing$. This completes the proof. 
Proposition. Let $K$ be a locally ordered $n$-complex, and let $f: K \rightarrow R^{p}$ be a moment map. Then

$$
\mathscr{B}(f)=c_{2 n-p-1}(K) \text {. }
$$

Proof. Let $\omega$ be a $(2 n-p-1)$-simplex of $K$, and let $\sigma$ and $\tau$ be $n$-simplices in the star of $\omega$. We must show that $f\left(\sigma^{\circ}\right) \cap f\left(\tau^{\circ}\right) \neq \varnothing$ if and only if $\sigma \cap \tau=\omega$ and either $\mu(\sigma, \tau)=1$ or $\mu(\tau, \sigma)=1$. Let $v_{1}, \ldots, v_{s}$ be the vertices of $\sigma$ and $\tau$ in the given order.

If $\sigma \cap \tau=\omega$ then each of $\sigma$ and $\tau$ has $n+1$ vertices, $2 n-p$ of which are repeated, so $s=p+2$. If $\mu(\sigma, \tau)=1$ we consider the two simplices $\sigma^{\prime}=$ $\left\langle v_{1}, v_{3}, v_{5}, \ldots\right\rangle, \tau^{\prime}=\left\langle v_{2}, v_{4}, v_{6}, \ldots\right\rangle$. The simplex $\sigma^{\prime}$ is a face of $\sigma$ and $\tau^{\prime}$ is a face of $\tau$. These simplices are disjoint and $\operatorname{dim} \sigma^{\prime}+\operatorname{dim} \tau^{\prime}=p$. By Shapiro's lemma, $f\left(\sigma^{\prime}\right) \cap f\left(\tau^{\prime}\right) \neq \varnothing$, so Lemmas 1 and 3 imply that $f\left(\sigma^{\circ}\right) \cap$ $f\left(\tau^{\circ}\right) \neq \varnothing$. We obtain the same conclusion if $\mu(\tau, \sigma)=1$.

Conversely, suppose that $f\left(\sigma^{\circ}\right) \cap f\left(\tau^{\circ}\right) \neq \varnothing$. By Lemma 1 ,

$$
\operatorname{dim} f\left(\sigma^{\circ}\right) \cap f\left(\tau^{\circ}\right)=2 n-p
$$

and since $\sigma \cap \tau \supset \omega$, we have

$$
\begin{aligned}
2 n-p & =\operatorname{dim}(f(\sigma) \cap f(\tau))>\operatorname{dim} f(\sigma \cap \tau) \\
& \geqslant \operatorname{dim} \omega=2 n-p-1 .
\end{aligned}
$$

It follows that $\operatorname{dim} f(\sigma \cap \tau)=\operatorname{dim} \omega$ so $\sigma \cap \tau=\omega$. So Lemmas 1 and 3 imply that there are faces $\sigma^{\prime}<\sigma$ and $\tau^{\prime}<\tau$ with $\sigma^{\prime} \cap \tau^{\prime}=\varnothing$, $\operatorname{dim} \sigma^{\prime}+$ $\operatorname{dim} \tau^{\prime}=p$, and $f\left(\left(\sigma^{\prime}\right)^{\circ}\right) \cap f\left(\left(\tau^{\prime}\right)^{\circ}\right) \neq \varnothing$. By Shapiro's lemma, the vertices of $\sigma^{\prime}$ and $\tau^{\prime}$ alternate along the moment curve, so $\mu(\sigma, \tau)=1$ or $\mu(\tau, \sigma)=1$.

This Proposition and Theorem 2 imply Theorem 1.

4. Questions. The proposition and the proof of Theorem 2 show that if $K$ is any finite purely $n$-dimensional complex then $c_{n-i}(K)$ is a cycle which represents $\mathrm{Sq}^{i}[K] \in H_{n-i}(K, \partial K)$. Our combinatorial definition of $c_{n-i}(K)$ is very similar to Steenrod's original definition of $\mathrm{Sq}^{i}$ in cohomology using cup- $i$ products [11]. Can Theorem 1 be proved directly using this relationship?

Our formula for the normal class $\bar{w}$ is also similar to Goldstein and Turner's formula [3] for the tangential class $w$. Can this relation be used to give a combinatorial proof of the Whitney duality theorem $w \cup \bar{w}=1$, or something more general?

\section{REFERENCES}

1. T. Banchoff, Integral normal classes for polyhedral surfaces in 4-space (to appear).

2. Stiefel-Whitney homology classes and singularities of projections for polyhedral manifolds, Proc. Sympos. Pure Math., vol. 27, Amer. Math. Soc., Providence, R. I., 1975, pp. 333-347.

3. R. Goldstein and E. Turner, A formula for Stiefel-Whitney homology classes, Proc. Amer. Math. Soc. 58 (1976), 339-342.

4. S. Halperin and D. Toledo, Stiefel-Whitney homology classes, Ann. of Math. (2) 96 (1972), 511-525. 
5. R. Hardt and C. McCrory, Steenrod operations in subanalytic homology, Compositio Math. (to appear).

6. N. Levitt and C. Rourke, The existence of combinatorial formulae for characteristic classes (to appear).

7. C. McCrory, Euler singularities and homology operations, Proc. Sympos. Pure Math., vol. 27, Amer. Math. Soc., Providence, R. I., 1975, pp. 371-380.

8. Geometric homology operations, Advances in Math. (to appear).

9. C. McCrory, D. Damiano and J. Stormes, Lectures on homology operations, Brown University, Providence, R. I., 1977.

10. A. Shapiro, Obstructions to the imbedding of a complex in a Euclidean space, Ann. of Math. (2) 66 (1957), 256-269.

11. N. Steenrod, Products of cocycles and extensions of mappings, Ann. of Math. (2) 48 (1947), $290-316$.

Department of Mathematics, Brown University, Providence, Rhode Island 02912 